\title{
Resultados do tratamento cirúrgico das fraturas do rádio distal: estudo clínico com técnica de osteossíntese percutânea e minimamente invasiva
}

\section{Clinical results of surgical treatment of distal radius fractures: a clinical study with minimally invasive percutaneous osteosynthesis}

Marcio Aurélio Aita ${ }^{1}$, Douglas Hideki Ikeuti ${ }^{1}$, Rodrigo Toledo Mota ${ }^{1}$, Guilherme Bonadia Bueno de Moraes ${ }^{1}$,

Marcos Vinícius Credidio ${ }^{1}$, Eduardo Fernandes da Costa ${ }^{1}$, Fernando Luvizotto Carvalho'

${ }^{1}$ Faculdade de Medicina do ABC (FMABC) - Santo André (SP), Brasil.

DOI: http://dx.doi.org/10.7322/abcshs.v39i1.255

\begin{abstract}
RESUMO
Introdução: A utilização de técnicas minimamente invasivas para o tratamento de fraturas do rádio distal teve um aumento significativo nos últimos três anos. Isso é explicado devido ao aumento na incidência de fraturas em pacientes economicamente ativos e à necessidade destes retornarem precocemente ao trabalho. Objetivo: Analisar os resultados clínico-funcionais dos pacientes com diagnóstico de fratura com desvio redutível e instável da extremidade distal do rádio submetidos ao tratamento cirúrgico pela técnica de osteossíntese percutânea e minimamente invasiva utilizando haste intramedular bloqueada. Métodos: Foram avaliados 53 pacientes submetidos ao tratamento cirúrgico da fratura da extremidade distal do rádio, redutível e instável, classificadas segundo Rayhack como Tipo IIB, pelo método minimamente invasivo, com haste intramedular bloqueada no período entre janeiro de 2011 e dezembro de 2012. O tempo de seguimento foi de seis a 24 meses, avaliando periodicamente parâmetros radiográficos, dor, medida do arco de movimento, força de preensão palmar e qualidade de vida através do questionário DASH. Resultados: Através da análise dos valores obtidos observamos uma melhora estatisticamente significante em todos os parâmetros clínicos analisados. O grau de força de preensão palmar e a medida do arco de movimento apresentaram melhora importante desde a terceira semana de pós-operatório, assim como a escala de dor e a avaliação do questionário DASH. Conclusão: A técnica minimamente invasiva é eficaz e segura, com melhora clínica e funcional e apresenta resultados superiores quando comparada a outros métodos descritos na literatura.
\end{abstract}

Palavras-chave: procedimentos cirúrgicos minimamente invasivos; fixação de fratura; fixação intramedular de fraturas.

\begin{abstract}
Introduction: The use of minimally invasive techniques for the treatment of the distal radius fractures has been increasing significantly over the past three years. This increase can be explained by the rising incidence of fractures in economically active patients and their need to early return to work activities. Objective: To analyze the clinical and functional results of patients diagnosed with distal radius fracture with reducible and unstable deviation, who underwent surgical treatment by the minimally invasive percutaneous osteosynthesis technique using a locked intramedullary nail. Methods: We evaluated 53 patients who underwent surgical treatment of reducible and unstable distal radius fractures, classified according to Rayhack as type IIB, using the minimally invasive method with intramedullary locked nail in the period between January 2011 and December 2012. The follow-up time was 6 to 24 months, periodically evaluating radiographic parameters, pain, range of motion, grip strength and quality of life through the DASH questionnaire. Results: Through the analysis of data obtained, we observed a statistically significant improvement in all clinical parameters analyzed. The degree of grip strength and range of motion presented an important improvement since the third week of the postoperative period, so did the pain scales and evaluation through the DASH questionnaire. Conclusion: The minimally invasive technique is a safe and effective procedure, presenting clinical and functional improvement and presenting superior results when compared to other methods described on the literature.
\end{abstract}

Keywords: surgical procedures, minimally invasive; fracture fixation; fracture fixation, intramedullary. 


\section{INTRODUÇÃO}

As fraturas que acometem o rádio distal estão entre as mais frequentes dentre aquelas que atingem o membro superior, representando 74,5\% das fraturas de antebraço com incidência de uma em cada dez mil pessoas ${ }^{1}$, sendo mais prevalente o acometimento dos membros dominantes ${ }^{2}$.

Atualmente a fratura da extremidade distal do rádio é vista como complexa, de prognóstico dependente do tipo de fratura e do tratamento adotado. Na busca por melhores resultados clínicos e funcionais, o método cirúrgico é cada vez mais indicado, principalmente pela evolução apresentada pelos implantes desenvolvidos especialmente para a extremidade distal do rádio. Apesar dos avanços tecnológicos, ainda há espaço para o tratamento conservador clássico, principalmente nas fraturas sem desvio ${ }^{3}$.

Nos últimos anos, o método cirúrgico evoluiu com o surgimento de técnicas de osteossíntese minimamente invasivas e abordagem com menor agressão do invólucro de partes moles, considerada uma abordagem mais biológica e com melhor prognóstico de recuperação do trauma cirúrgico ${ }^{4}$.

Os implantes mais utilizados no tratamento destas fraturas são os fios de Kirschner, a placa volar bloqueada (que apresenta os melhores resultados), sugeridos nas últimas revisões sistemáticas e o fixador externo transarticular ${ }^{4}$. Além desses métodos consagrados na literatura há ainda novos implantes desenvolvidos para o tratamento cirúrgico das fraturas da extremidade distal do rádio, como a haste intramedular bloqueada ${ }^{5}$, que pode ser utilizada de maneira percutânea ${ }^{6}$.

A possibilidade da redução incruenta e indireta desta fratura, sem visibilizar o foco fraturário, nos permite uma reabilitação cada vez mais precoce, pois além da menor agressividade do método há maior estabilidade pós-operatória ${ }^{6}$.

Tendo em vista que não existem resultados estatísticos significantes evidentes nos trabalhos publicados até o momento abordando o tratamento cirúrgico das fraturas da extremidade distal do rádio, se somado ao fato das metanálises realizadas por Handoll e Madhok ${ }^{7,8}$ citarem a necessidade da realização de pesquisas com melhor evidência qualitativa, decidimos realizar este estudo utilizando a técnica minimamente invasiva com haste intramedular bloqueada.

O objetivo deste estudo é analisar os resultados clínico-funcionais (força de preensão palmar, arco de movimento do punho e dor) dos pacientes com diagnóstico de fratura extra-articular, com desvio redutível e instável classificadas como tipo IIB de Rayhack ${ }^{9}$, da extremidade distal do rádio submetidos ao tratamento cirúrgico pela técnica de osteossíntese percutânea e minimamente invasiva, utilizando como implante a haste intramedular bloqueada. Além disso, foram avaliados os resultados quanto à melhora da qualidade de vida através da aplicação do questionário Disabilities of the Arm, Shoulder and Hand (DASH) ${ }^{10}$.

\section{MÉTODOS}

Foram avaliados 96 pacientes, no período de janeiro de 2011 a dezembro de 2012, atendidos nos ambulatórios do Grupo da Mão e Microcirurgia da Faculdade de Medicina do ABC, localizado em Santo André, São Paulo, com diagnóstico de fratura da extremidade distal do rádio, sendo que 53 pacientes foram incluídos no estudo e submetidos a exame físico e radiografias simples nas incidências póstero-anterior (PA) e perfil (P) do membro acometido (Figura 1).

Foram incluídos no estudo os pacientes adultos de 19 a 69 anos, de ambos os sexos, com diagnóstico clínico e por imagem de fratura da extremidade distal do rádio do tipo IIB segundo Rayhack e que preencheram o Termo de Consentimento Voluntário, Livre e Esclarecido e o protocolo de conflito de interesses, conforme recomendação do Comitê de Ética em Pesquisa.

Foram excluídos os pacientes com doenças associadas no punho, como as doenças osteometabólicas, ou que foram submetidos a qualquer procedimento cirúrgico prévio.

A avaliação funcional foi realizada por profissionais do Setor de Terapia Ocupacional de Mão do Hospital Estadual Mário Covas, Santo André, São Paulo.

Os pacientes foram avaliados quanto a dor, qualidade de vida, força e arco de movimento em três momentos: na terceira e sexta semana do pós operatório e no último atendimento antes da alta do ambulatório.

A mensuração funcional foi executada pela medida (em graus) dos arcos de movimento do punho (ADM) com goniômetro único e específico. A mensuração da medida da força de preensão palmar foi feita em quilograma-força (kgf) com o dinamômetro hidráulico Jamar ${ }^{\circledR}$.

A análise clínica da dor foi realizada pela escala Visual Analogue Scale (VAS), de zero até dez, para a avaliação subjetiva.

A avaliação da qualidade vida foi realizada através da aplicação do questionário $\mathrm{DASH}^{10}$, instrumento utilizado na maioria dos estudos publicados para o tratamento da fratura da extremidade distal do rádio.

Foram avaliados também o membro dominante mais acometido pela fratura, índice de complicações após o terceiro e o sexto mês e tempo de seguimento de cada paciente.

Os pacientes foram submetidos à osteossíntese minimamente invasiva e percutânea, utilizando-se haste intramedular bloqueada $a^{5,11}$ (Figura 2), conforme descrito a seguir:

1. Realiza-se redução incruenta da fratura e estabiliza-se provisoriamente com um ou dois fios de Kirschner inseridos na região da ulna distal até a coluna medial do rádio, com o auxílio da radioscopia.

2. A via de acesso é mínima, dorsal e radial de $2 \mathrm{~cm}$, utilizando o primeiro túnel extensor como parâmetro anatômico. A abertura da retinácula dorsal é longitudinal e única, com exposição dos tendões extensores do primeiro túnel, que são afastados dorsalmente.

3. Neste momento é implantado um fio de Kirschner padrão e guia na região proximal ao estiloide do rádio, encavilhando-o na região intramedular metafisária do rádio, logo abaixo do primeiro túnel extensor.

4. Executa-se a fresagem, com broca específica e canulada, do rádio utilizando o fio guia como parâmetro, obrigatoriamente. 


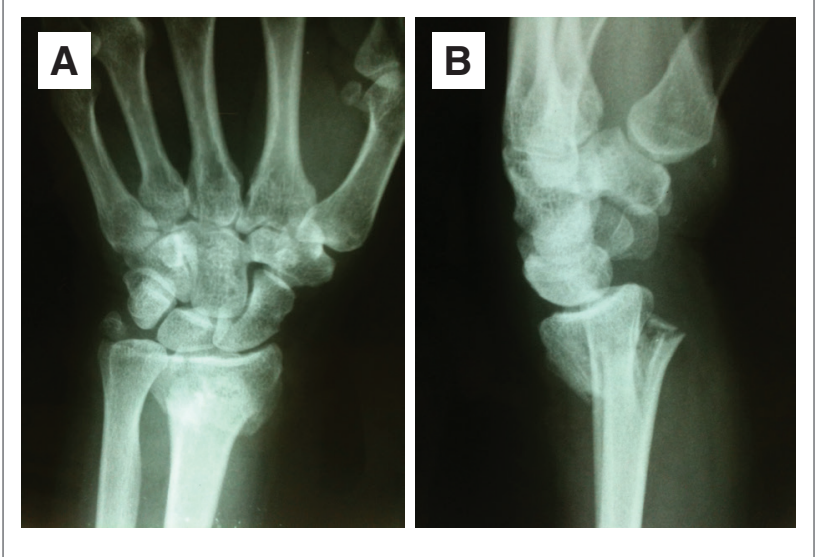

Figura 1: Radiografia pré-operatória. (A) póstero-anterior; (B) em perfil

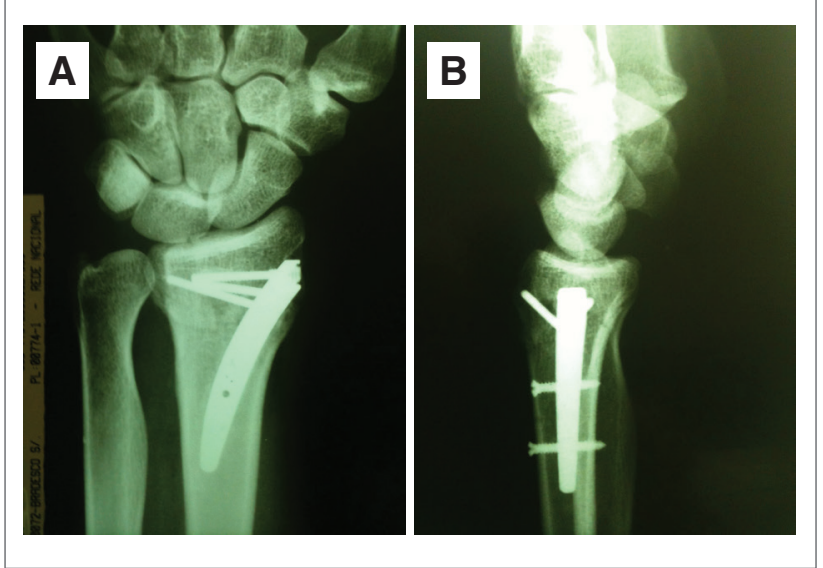

Figura 2: Radiografia pós-operatória. (A) póstero-anterior; (B) em perfil

5. Realiza-se, então, a colocação manual de fresas com os tamanhos da haste (variando de uma a cinco), e quando há um ajuste perfeito desta fresa com a medular do rádio, encontra-se o tamanho da haste a ser utilizada.

6. Posiciona-se a haste intramedular com o auxílio da radioscopia.

7. Faz-se a estabilização distal da fratura, com três parafusos bloqueados laterais de 2,7 $\mathrm{mm}$, permitindo a estabilidade angular, utilizando o guia que está preso à haste.

8. Estabiliza-se proximalmente o sistema com dois parafusos corticais dorsais de 2,4 mm, com auxílio do guia específico.

9. Suturam-se os planos e a pele.

10. Realiza-se radioscopia e radiografia pós-operatória do punho para controle pós-cirúrgico.

11. Confecciona-se curativo para o punho.

Os pacientes foram avaliados antes da cirurgia e depois, clínica e radiograficamente, na terceira e sexta semanas, e no último atendimento. Todos foram reabilitados pelo Setor De Terapia Ocupacional de Mão da mesma instituição, com o mesmo protocolo para analgesia e cinesioterapia desde a primeira semana de pós-operatório até a alta do setor.

Este estudo foi aprovado pela Comissão de Ética e Pesquisa Médica da Faculdade de Medicina do ABC em março de 2007.

\section{Análise estatística}

A fim de analisar dados quantitativos (idade, tempo de seguimento, escala de dor, avaliação do DASH, medida da força de preensão e do arco de movimento, tempo de seguimento) foi utilizada a média como medida de tendência central e a medida de dispersão adotada foi o desvio padrão.

Nas análises estatísticas adotamos o nível de significância de 5\% $(\mathrm{p}=0,05)$ para a aplicação dos testes estatísticos, ou seja: quando o valor $\mathrm{p}$ for menor do que $5 \%$, temos uma diferença estatisticamente significante (no caso de comparações), ou uma relação estatisticamente significante (no caso de relacionamentos), isto é, encontramos uma efetiva diferença (no caso de comparações), ou uma relação forte (no caso de relacionamentos). Quando o valor $\mathrm{p}$ for igual ou maior do que 5\%, temos uma diferença estatisticamente não significante (no caso de comparações), ou uma relação estatisticamente não significante (no caso de relacionamentos), isto é, encontramos uma semelhança (no caso de comparações), ou uma relação fraca (no caso de relacionamentos).

Foi utilizada a planilha eletrônica MS-Excel, em sua versão do MSOffice 2010, para a organização dos dados, e o pacote estatístico IBM SPSS (Statistical Package for the Social Sciences), em sua versão 21.0, para a obtenção dos resultados.

\section{RESULTADOS}

Os pacientes incluídos no estudo tinham idade variando entre 19 e 69 anos, correspondendo a uma média de 36,5 anos e desvio padrão (DP) de 13,59. O tempo de seguimento variou de seis a 23 meses, sendo o tempo médio de 13,4 meses (DP 5,32) e 52 pacientes (98\%) mantiveram a redução inicial obtida e a consolidação da fratura.

Dentre os pacientes, 26 eram do sexo feminino $(49,1 \%)$ e 27 do masculino (50,9\%). Somente quatro pacientes $(7,5 \%)$ retornaram ao trabalho antes da terceira semana. Um total de 46 pacientes $(86,8 \%)$ retornaram antes da sexta semana e três pacientes $(5,7 \%)$ tiveram o seu retorno somente depois do sexto mês.

$\mathrm{O}$ arco de movimento (Figura 3) na terceira semana variou de $160 \mathrm{a}$ 230, apresentando média de 213,08 (DP 15,87). Na sexta semana este se elevou, variando de 200 a 230, com média de 220,43 (DP 6,15). No sexto mês este variou de 215 a 239, com média de 222,17 (DP 4,21).

A força de preensão palmar (Figura 4) na terceira semana variou de 5 a 20, apresentando média de 11,94 (DP 2,93). Na sexta semana este se elevou, variando de 9 a 26 com média de 17,34 (DP 3,43). No sexto mês este variou de 12 a 26 possuindo média de 19,06 (DP 3,35).

A avaliação da qualidade de vida por meio do questionário DASH (Figura 5) obteve média de 22,24 (DP 11,67) na terceira semana, variando de 6 a 61 . Já na sexta semana este apresentou uma média de 8,47 (DP 7,87), variando de 1 a 36. Por fim no sexto mês a média foi de 1,34 (DP 1,45), variando de 1 a 9.

A análise clínica da dor realizada pela escala VAS (Figura 6) variou de 0 a 9 na terceira semana, possuindo média de 2,3 (DP 1,87). Na sexta semana esta obteve média de 1,26 (DP 1,11), variando de 0 a 6 . Já no sexto mês a média foi de 0,15 (DP 0,63), variando de 0 a 3 . 


\section{DISCUSSÃO}

A evolução no tratamento da fratura da extremidade distal do rádio ocorre em dois aspectos: a tecnológica, com desenvolvimento de implantes que permitem a estabilidade angular, e a biológica, que igualmente estabiliza o osso, mas preserva o invólucro de partes moles e, assim, permite o retorno funcional mais precoce dessa articulação, proporcionando ao paciente menor, danos econômicos e sociais.

O novo design dos implantes desenvolvidos para a extremidade distal do rádio, bem como o instrumental, como os guias externos acoplados e a utilização de materiais biocompatíveis, estabelecidos nos últimos dez anos, possibilitam a técnica minimamente invasiva.

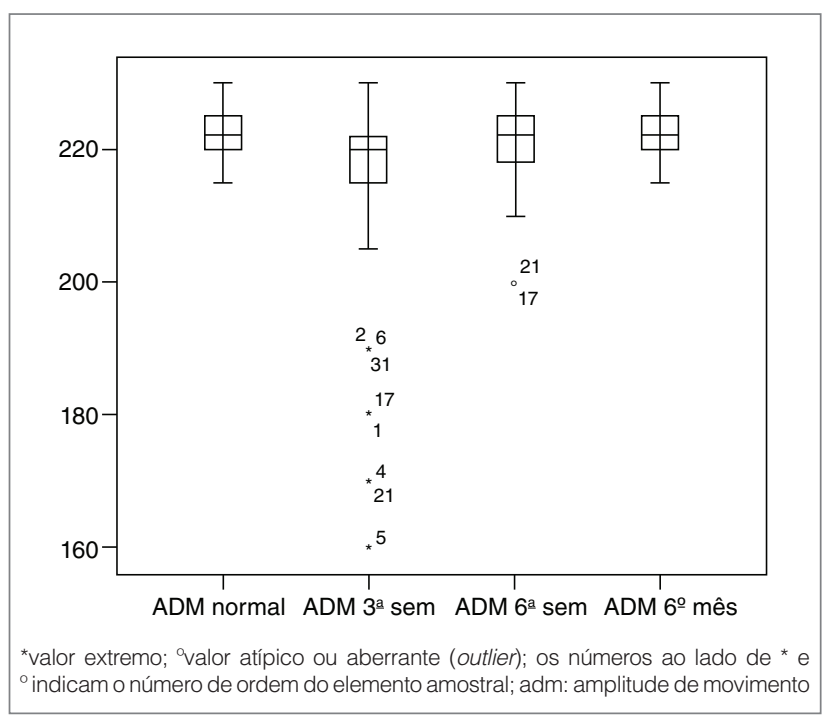

Figura 3: Representação gráfica da média com desvio padrão do arco de movimento (ADM) analisado na terceira e na sexta semanas e no sexto mês

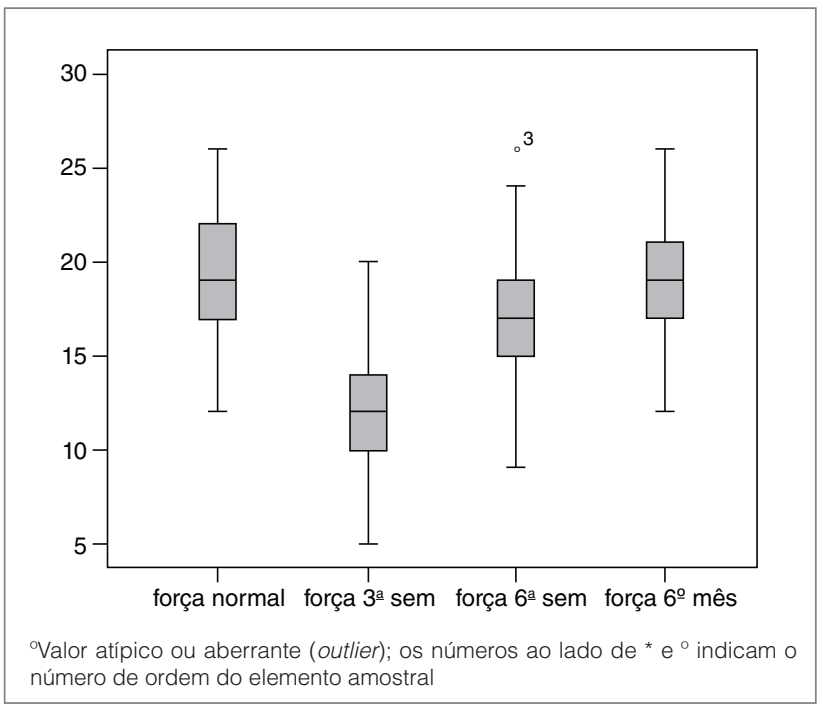

Figura 4: Representação gráfica da média com desvio padrão da força de preensão palmar analisada na terceira e na sexta semanas e no sexto mês
Ao analisarmos os parâmetros radiográficos, $98 \%$ dos pacientes mantiveram a redução inicial obtida da fratura, mostrando que o implante e o princípio de osteossíntese utilizado neste estudo são seguros e estáveis, permitindo a consolidação óssea da fratura de maneira eficaz.

Quando comparamos horizontalmente os resultados clínico-funcionais (arco de movimento e força de preensão palmar), observamos melhora desde a terceira semana após a cirurgia. Os valores do questionário de qualidade de vida e da escala de dor também apresentaram valores superiores, em todos os momentos do estudo.

Os dados obtidos neste estudo também foram comparados com os trabalhos publicados na literatura ${ }^{11-15}$. Obtivemos resultados

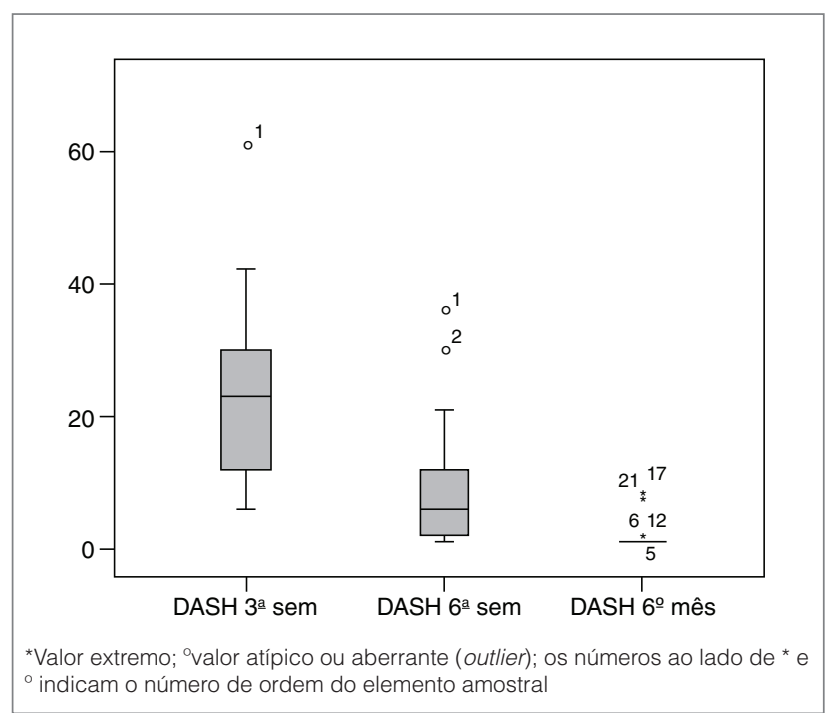

Figura 5: Representação gráfica da média com desvio padrão da avaliação de qualidade de vida por meio do questionário DASH analisada na terceira e na sexta semanas e no sexto mês

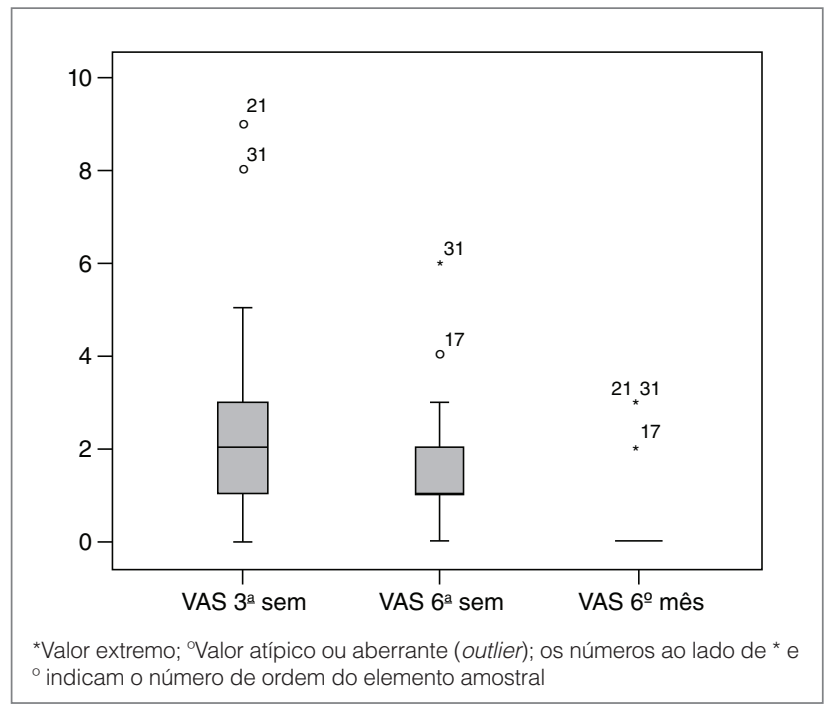

Figura 6: Representação gráfica da média com desvio padrão da análise clínica da dor realizada pela Escala Analógica Visual (VAS) analisada na terceira e na sexta semanas e no sexto mês 
semelhantes àqueles da pesquisa de Schonemann et al. ${ }^{11}$, com melhores resultados de força de preensão palmar para os pacientes tratados com haste intramedular em relação aos tratados com fixador externo, e resultados semelhantes nos parâmetros radiográficos analisados, sem perda da redução inicial, após a consolidação da fratura. Quando comparamos nossos valores com o estudo de Cui et al. ${ }^{16}$, verificamos semelhanças nos resultados, com evidências melhores para os pacientes de nosso protocolo tratados com haste em relação aos tratados com placa volar e fixador externo.

Embora o estudo de Zenke et al. ${ }^{17}$ não mostre diferenças significativas entre o método convencional e o minimamente invasivo, com o uso da placa volar, no tratamento das fraturas da extremidade distal do rádio, observamos melhores resultados clínico-funcionais e do questionário DASH em nosso estudo em relação à pesquisa de
Orbay et al. ${ }^{18}$, como a força de preensão palmar de $98 \%$ versus $77 \%$ e DASH de 1,2 versus 8,28.

Ao avaliar as complicações nos estudos de Richard et al. ${ }^{19} \mathrm{e}$ Rampoldi e Marsico ${ }^{20}$, ambas as publicações apresentaram resultados inferiores aos do nosso estudo.

Embora não seja comum em nosso meio o uso da haste intramedular para o rádio em sua extremidade distal, pudemos observar que a curva de aprendizado é pequena, e que os resultados obtidos neste estudo foram satisfatórios e promissores.

Conclui-se que a técnica minimamente invasiva é eficaz e segura, com melhora clínica e funcional em todos os momentos do estudo. O princípio de osteossíntese e o implante utilizado são estáveis.

Há superioridade dos resultados clínico-funcionais (grau de força e arco de movimento), DASH e VAS, quando comparados a outros métodos descritos na literatura.

\section{REFERÊNCIAS}

1. Alffram PA, Bauer GC. Epidemiology of fractures of the forearm. A biomechanical investigation of bone strength. J Bone Joint Surg Am. 1962;44-A:105-14.

2. Angelini LC, Grecco MAS. Tratamento da pseudartrose do terço distal do rádio. Acta Ortop Bras. 2005;13(2):95-99. http://dx.doi.org/10.1590/S1413-78522005000200006

3. Fernandez DL, Palmer AK. Fractures of the distal radius. In: Green DP, Hotchkiss RN, Pederson WC, editors. Green's operative hand surgery. 4th ed. New York: Churchill Livingstone; 1999. p. 925-85.

4. Wei DH, Poolman RW, Bhandari M, Wolfe VM, Rosenwasser MP. External fixation versus internal fixation for unstable distal radius fractures: a systematic review and meta-analysis of comparative clinical trials. J Orthop Trauma. 2012;26(7):386-94. http://dx.doi.org/10.1097/BOT.0b013e318225f63c

5. Nishiwaki M, Tazaki K, Shimizu H, llyas AMJ. Prospective study of distal radial fractures treated with an intramedullary nail. Bone Joint Surg Am. 2011;93(15):1436-41 http://dx.doi.org/10.2106/JBJS.J.01159

6. Sen MK, Strauss N, Harvey EJ. Minimally invasive plate osteosynthesis of distal radius fractures using a pronator sparing approach. Tech Hand Up Extrem Surg. 2008;12(1):2-6. http://dx.doi.org/10.1097/BTH.0b013e3180cac281

7. Handoll HH, Madhok R. WITHDRAWN: surgical interventions for treating distal radial fractures in adults. Cochrane Database Syst Rev. 2009;(3):CD003209.

http://dx.doi.org/10.1002/14651858.CD003209.pub2

8. Handoll HH, Madhok R. Surgical interventions for treating distal radial fractures in adults. Cochrane Database Syst Rev. 2003;(3):CD003209. http://dx.doi.org/10.1002/14651858.CD003209

9. Rayhack JM. The history and evaluation of percutaneous pinning of displaced distal radius fractures. Orthop Clin North Am. 1993;24(2):287-300

10. Jester A, Harth A, Wind G, Germann G, Sauerbier M. Disabilities of the arm, shoulder and hand (DASH) questionnaire: determining functional activity profiles in patients with upper extremity disorders. J Hand Surg Br. 2005;30(1):23-8.

http://dx.doi.org/10.1016/j.jhsb.2004.08.008

11. Schønnemann JO, Hansen TB, Søballe K. Randomised study of non-bridging external fixation compared with intramedullary fixation of unstable distal radial fractures. J Plast Surg Hand Surg. 2011;45(4-5):232-7

http://dx.doi.org/10.3109/2000656X.2011.613243

12. Mehling I, Müller LP, Delinsky K, Mehler D, Burkhart KJ, Rommens PM. Number and locations of screw fixation for volar fixed-angle plating of the distal radius fracture: biomechanical study. J Hand Surg Am. 2010;35(6):885-91.

http://dx.doi.org/10.1016/j.jhsa.2010.03.027

13. McQueen MM. Redisplaced unstable fractures of the distal radius. A randomised, prospective study of bridging versus non-bridging external fixation. J Bone Joint Surg Br. 1998;80(4):665-9.

14. Wolfe SW, Austin G, Lorenze M, Swigart CR, Panjabi MM. A biomechanical comparison of different wrist external fixators with and without K-wire augmentation. J Hand Surg Am. 1999; 24(3):516-24.

http://dx.doi.org/10.1053/jhsu. 1999.0516

15. Yamako G, Ishii Y, Matsuda Y, Noguchi H, Hara T. Biomechanical characteristics of nonbridging external fixators for distal radius fractures. J Hand Surg Am. 2008;33(3):322-6. http://dx.doi.org/10.1016/j.jhsa.2007.09.019

16. Cui Z, Pan J, Yu B, Zhang K, Xiong X. Internal versus external fixation for unstable distal radius fractures: an up-to-date metaanalysis. Int Orthop. 2011;35(9):1333-41. http://dx.doi.org/10.1007/s00264-011-1300-0

17. Zenke $Y$, Sakai A, Oshige T, Moritani S, Fuse Y, Maehara T, et al Clinical results of volar locking plate for distal radius fractures: conventional versus minimally invasive plate osteosynthesis. J Orthop Trauma. 2011;25(7):425-31. http://dx.doi.org/10.1097/BOT.0b013e3182008c83

18. Orbay JL, Badia A, Indriago IR, Infante A, Khouri RK, Gonzales E, et al. The extended flexor carpi radialis approach: a new perspective for the distal radius fracture. Tech Hand Up Extrem Surg. 2001;5(4):204-11

19. Richard MJ, Wartinbee DA, Riboh J, Miller M, Leversedge FJ Ruch DS. Analysis of the complications of palmar plating versus external fixation for fractures of the distal radius. J Hand Surg Am. 2011;36(10):1614-20

http://dx.doi.org/10.1016/j.jhsa.2011.06.030

20. Rampoldi M, Marsico S. Complications of volar plating of distal radius fractures. Acta Orthop Belg. 2007;73(6):714-9. 